\title{
Researchers promise to be more open about use of animals in their work
}

In the seventh paragraph of this News story, we incorrectly listed "the Association of Medical Research Councils" as one of the signatories to a declaration about the use of animals in research (BMJ 2012;345:e7101, doi:10.1136/bmj.e7101). The correct name of the organisation that has signed up is, in fact, the Association of Medical Research Charities.

Cite this as: BMJ 2012;345:e7161

๑ BMJ Publishing Group Ltd 2012 